\title{
Caracterização do consumo alimentar de lactentes paulistas com idade entre seis e doze meses
}

\author{
Characterization of the food consumption of breastfed infants \\ between six to twelve months of age in the State of São Paulo
}

Adriana Passanha (https://orcid.org/0000-0001-6659-1251) ${ }^{1}$

Maria Helena D’Aquino Benício (https://orcid.org/0000-0003-1851-1178) ${ }^{1}$

Sonia Isoyama Venancio (https://orcid.org/0000-0001-7147-3292) ${ }^{2}$

${ }^{1}$ Faculdade de Saúde Pública, Universidade de São Paulo, Departamento de Nutrição. Av. Dr. Arnaldo 715, Cerqueira César. 01246-904 São Paulo SP Brasil.adriana.passanha@ gmail.com

${ }_{2}^{2}$ Instituto de Saúde, Secretaria de Estado da Saúde de São Paulo. São Paulo SP Brasil.

\begin{abstract}
The scope of this article is to characterize the food consumption of breastfed infants living in the State of São Paulo during the second semester of life. It is a cross-sectional study with a representative sample of 14,327 infants from six to twelve months of age resident in 76 municipalities in the State of São Paulo in 2008. The characterization of infants, mothers and each municipality was conducted. Besides the evaluation of food products and dishes consumed, indicators based on the recommendations of the World Health Organization, the Brazilian Ministry of Health and studies on infant feeding practices were calculat$e d$. The analysis of food consumption according to age bracket (6|-8.9 months and 9|-12 months) and to breastfeeding pattern was performed using the chi-square test, considering a $p<0.05$ significance level. The majority of infants were being breastfed (56.1\%). The consumption of porridge, water, fruit, salty pap, meat and unhealthy foods was more prevalent among older infants and those not breastfed. The majority of breastfed infants revealed a negative classification for minimum dietary diversity (68.2\%), dietary adequacy (72.7\%) or minimum acceptable diet $(71.1 \%)$. The feeding of breastfed infants from the State of São Paulo falls short of the recommendations prescribed by the authorities.
\end{abstract}

Key words Food consumption, Breastfed infant, Public health
Resumo O objetivo deste artigo é caracterizar o consumo alimentar de lactentes paulistas no segundo semestre de vida. Estudo transversal, com amostra representativa de 14.327 lactentes de seis a doze meses, residentes de 76 municípios do estado de São Paulo, em 2008. Foi realizada a caracterização da amostra de lactentes, de mães e de cada município estudado. Em relação ao consumo alimentar, além da avaliação sobre alimentos e preparações consumidos, foram calculados indicadores com base nas recomendações da Organização Mundial da Saúde, Ministério da Saúde e estudos sobre práticas de alimentação infantil. A análise do consumo alimentar segundo faixa etária (6|-8,9 meses e $9 \mid-12$ meses) e padrão de aleitamento materno utilizou o teste de qui-quadrado, considerando-se significante $p<0,05$. A maioria dos lactentes encontrava-se em aleitamento materno (56,1\%). O consumo de mingau, água, fruta, papa salgada, carne e de alimentos não saudáveis foi mais prevalente nos lactentes mais velhos e naqueles não amamentados. A maioria dos lactentes apresentou classificação negativa para diversidade mínima (68,2\%), adequação da dieta (72,7\%) ou dieta minimamente aceitável (71,1\%). A alimentação dos lactentes paulistas encontra-se aquém das recomendações preconizadas pelas autoridades.

Palavras-chave Consumo de alimentos, Lactente, Saúde pública 


\section{Introdução}

Os dois primeiros anos de vida são caracterizados por crescimento e desenvolvimento acelerados ${ }^{1}$, sendo a alimentação essencial nesses processos ${ }^{2,3}$. A demanda nutricional do lactente é atendida pela amamentação exclusiva até o sexto mês. Após esse período outros alimentos são necessários, mas recomenda-se manter o aleitamento materno até os dois anos de idade ou mais ${ }^{2,3}$.

Uma alimentação complementar saudável inclui, dentre diversos aspectos, alimentos introduzidos em momento oportuno, adequados em água, energia e nutrientes, sem excesso de sal, gordura, ou açúcar, e em quantidade e consistência apropriadas ${ }^{1,4,5}$. Condutas alimentares inadequadas nessa fase podem trazer diversas consequências negativas, como retardo de crescimento, atraso intelectual, anemia, aumento de peso e, a longo prazo, desenvolvimento de doenças crônicas não transmissíveis ${ }^{1,4,6-9}$. Nesse período também formam-se hábitos alimentares que permearão até a fase adulta ${ }^{5}$.

Dados do Sistema de Vigilância Alimentar e Nutricional (SISVAN) apontam que, em 2008, a prevalência de risco de sobrepeso entre lactentes paulistas foi de $19,0 \%$, enquanto as prevalências de sobrepeso e obesidade foram, respectivamente, de $6,7 \%$ e $7,2 \%$; em 2015, tais valores permaneceram os mesmos ${ }^{10}$. Embora a cobertura do SISVAN no estado de São Paulo de modo geral seja baixa, esses achados são preocupantes, pois englobam quase um terço dos lactentes paulistas acompanhados por este Sistema e, possivelmente, refletem inadequações de seu consumo alimentar.

A importância da alimentação complementar adequada e saudável para o pleno desenvolvimento infantil é inquestionável. Assim, o objetivo deste estudo foi caracterizar o consumo alimentar de lactentes paulistas no segundo semestre de vida.

\section{Métodos}

\section{Delineamento e população de estudo}

Estudo transversal, com base nas informações referentes aos municípios paulistas participantes da Pesquisa de Prevalência de Aleitamento Materno (PPAM) em Municípios Brasileiros, a qual teve como objetivo verificar a situação do aleitamento materno e da alimentação complementar no Brasil. Os dados foram coletados durante a Campanha Nacional de Vacinação de
$2008^{11}$.A PPAM incluiu, em sua amostra, crianças menores de um ano - detalhes sobre cálculo do tamanho amostral podem ser obtidos em outras publicações ${ }^{12,13}$; para o presente estudo, foram incluídos lactentes de seis meses completos a doze meses incompletos - optou-se por não incluir menores de seis meses devido à recomendação de amamentação exclusiva até essa idade. No estado de São Paulo, participaram da PPAM, em 2008, 31528 lactentes menores de um ano residentes de 77 municípios, dos quais 14573 estavam na faixa etária de seis a 11,9 meses. Foram excluídos 246 lactentes sem informações sobre idade e sobre município de nascimento; ainda, foi excluído um município, pois no mesmo participaram apenas lactentes menores de seis meses. Assim, foram incluídos no presente estudo 14327 lactentes com 6|-12 meses de idade, residentes de 76 municípios do estado. A distribuição destes municípios está ilustrada na Figura 1.

\section{Caracterização dos lactentes e das mães}

Foram obtidas informações sobre características dos lactentes (faixa etária: em meses; sexo: masculino; feminino, peso ao nascer: $<2500 \mathrm{~g}$; $\geq 2500$ g, tipo de parto: cesárea; vaginal, local de acompanhamento ambulatorial: particular; Unidade Básica de Saúde tradicional; Programa Agentes Comunitários de Saúde (PACS)/ Estratégia Saúde da Família (ESF)) e das mães (faixa etária: 20 anos; de 20 a 35 anos; $\geq 35$ anos, paridade: primípara; multípara, situação de trabalho: trabalha fora; não trabalha fora, escolaridade em anos de estudo: $\leq 8 ; 9$ a 11; e $\geq 12$ ). Em relação ao trabalho materno, a categoria "Está sob Licença Maternidade" foi deixada como missing porque considerou-se que dificilmente lactentes com mais de seis meses teriam mães nesta condição. Apenas 115 (1,0\%) dos lactentes eram filhos destas mulheres; deixá-los como missing não provocou diferenças significantes na amostra (dados não apresentados).

\section{Caracterização dos municípios}

As informações sobre variáveis contextuais que caracterizaram os municípios (grau de urbanização; porte populacional; cobertura da ESF, do PACS e do SISVAN; Índice Paulista de Responsabilidade Social (IPRS)) foram obtidas dos sítios eletrônicos da Fundação Sistema Estadual de Análise de Dados ${ }^{14}$, da Secretaria de Estado da Saúde do Governo do Estado de São Paulo ${ }^{15}$, e do Departamento de Atenção Básica do Ministério 
Figura 1. Distribuição dos 76 municípios paulistas incluídos no estudo. Estado de São Paulo, 2008.

da Saúde ${ }^{10}$. A cobertura do SISVAN foi calculada segundo Enes et al. ${ }^{16}$. O IPRS identifica o desenvolvimento dos municípios nas dimensões renda, longevidade e escolaridade; considerando apenas a dimensão riqueza, os municípios são classificados em cinco grupos: os grupos 1 e 2 referem-se a municípios com alta riqueza, e os grupos 3,4 e 5 correspondem a municípios com baixa riqueza ${ }^{14}$. Considerando que as características dos lactentes e das mães foram coletadas em 2008, os dados contextuais também corresponderam ao ano de 2008.

\section{Caracterização do consumo alimentar}

A parte do questionário sobre consumo alimentar incluiu perguntas referentes ao dia anterior à pesquisa - o uso da "situação atual" (current status) é recomendado para descrever práticas de alimentação infantil e minimiza possíveis vieses de memória do informante ${ }^{17}$. Foi avaliado o consumo de: leite materno; outros tipos de leite (que não fosse o leite materno); mingau (doce ou salgado); água; chá; fruta (em pedaço ou amassada); suco ou água de coco natural; papa salgada (destaca-se que o termo "salgada" não teve como objetivo adjetivar a expressão ou induzir ao entendimento de que se refere a uma preparação com excesso de sal) ${ }^{1}$; carne; feijão; verduras ou legumes; suco industrializado ou água de coco em caixinha; refrigerante; café; e alimentos adoçados (com açúcar, mel, melado ou adoçante). Avaliouse também o uso de bicos artificiais: mamadeira ou chuquinha; e chupeta. As perguntas dessa parte do questionário eram fechadas, com três possibilidades de resposta: "sim", "não" ou "não sabe". Com estas informações, foram calculados diferentes indicadores com base nas recomendações da $\mathrm{OMS}^{17}$, Ministério da Saúde ${ }^{1}$ e estudos sobre alimentação infantil ${ }^{18,19}$, conforme descrito a seguir:

- Aleitamento materno: consumo de leite materno no dia anterior;

- Lácteos: consumo concomitante de leite materno e de outros tipos de leite;

- Bicos artificiais: uso isolado ou concomitante de mamadeira, chuquinha ou chupeta;

- Alimentos não saudáveis: consumo isolado ou concomitante de suco industrializado, água de coco em caixinha, refrigerante, café, alimentos adoçados, bolacha, biscoito ou salgadinho; 
- Alimentos ricos em ferro: consumo de carne ou de feijão ${ }^{17}$;

- Alimentação oportuna: consumo concomitante de fruta (em pedaço ou amassada) e de papa salgada, calculada apenas para lactentes de 6|-9 meses $^{19}$;

- Consistência adequada da papa salgada: havia quatro diferentes classificações de consistência da papa salgada no questionário: em pedaços, amassada, passada pela peneira ou liquidificada, sendo que mais de uma classificação poderia ser marcada como possível. Considerou-se a consistência da papa salgada adequada quando foi consumida "em pedaços", "amassada", ou quando estas duas alternativas foram assinaladas ${ }^{1}$;

- Densidade energética adequada: consumo de uma papa salgada com consistência adequada para lactentes de 6|-7 meses, e o consumo de duas papas salgadas na consistência adequada para aqueles com $7 \mid-12$ meses $^{19}$;

- Diversidade mínima da dieta: consumo concomitante de quatro grupos alimentares: "feijão", "carne", "outros tipos de leite" e "fruta (em pedaço ou amassada), verdura e legume"17. Ressalta-se que o consumo de suco natural não foi considerado como um alimento do grupo das frutas, porque no questionário a pergunta engloba tanto o consumo de suco natural quanto de água de coco, e porque sucos, mesmo naturais, não proporcionam os mesmos benefícios da fruta inteira: fibras e nutrientes podem ser perdidos durante o preparo, e o poder de saciedade do suco é inferior ${ }^{1}$;

- Adequação da dieta: combinação dos indicadores "consistência adequada da papa salgada" e "diversidade mínima da dieta"18;

- Dieta minimamente aceitável: combinação do consumo concomitante de todos os grupos alimentares avaliados ("feijão", "carne", "legume e verdura”, "fruta em pedaço ou amassada" e "lácteos") com densidade energética adequada ${ }^{19}$.

\section{Análise estatística}

Vale ressaltar que a PPAM-2008 é uma pesquisa feita com amostragem probabilística complexa e por isso requer procedimentos específicos para sua análise. Todas as análises do presente estudo levaram em consideração a estrutura complexa da amostra, com a utilização do comando $s v y$. Devido às diferenças populacionais entre os municípios estudados, cada plano correspondeu a uma fração amostral diferente, representada pelo tamanho estimado da amostra sobre o número de crianças a serem vacinadas. $\mathrm{O}$ inverso dessa fração foi aplicado como peso das crianças em cada município ${ }^{12}$. Para a análise descritiva, foram utilizadas frequências absolutas e relativas. A análise de consumo alimentar segundo faixa etária e padrão de aleitamento materno utilizou o teste de qui-quadrado, considerando-se significante $\mathrm{p}<0,05$. Os dados foram analisados no programa Stata/SE 13.1.

\section{Aspectos éticos}

Este estudo utilizou dados secundários e foi aprovado pelo Comitê de Ética da Faculdade de Saúde Pública da Universidade de São Paulo. No estudo primário, considerando a estratégia adotada de aplicação de um questionário rápido nas filas de vacinação, o Termo de Consentimento Livre e Esclarecido foi aplicado mediante consentimento verbal do responsável, respeitando-se o direto de recusa e interrupção da entrevista e garantindo o sigilo das informações ${ }^{12}$.

\section{Resultados}

A Tabela 1 mostra a caracterização da amostra de lactentes e mães. A proporção de lactentes que nasceram com baixo peso foi de $9,2 \%$, e mais de $60 \%$ realizavam acompanhamento ambulatorial em locais públicos. Aproximadamente 3/4 das mães tinham de 20 a 35 anos. Houve predomínio de mães com nove a 12 anos de estudo $(52,4 \%)$.

As características municipais são descritas na Tabela 2. Metade dos municípios tinha grau de urbanização de $90 \%$ e mais. A maior parte apresentou cobertura menor que $15 \%$ para ESF, mas maior que $70 \%$ para PACS, e a grande maioria estava com menos de $5 \%$ de cobertura do SISVAN. Apenas $1 / 3$ dos municípios foram classificados com alta riqueza (IPRS 1 ou 2).

A Tabela 3 mostra características do consumo alimentar e do uso de bicos artificiais em relação à faixa etária e à situação de aleitamento materno dos lactentes. A prevalência de amamentação foi de $56,1 \%$. A frequência de consumo de outros tipos de leite foi maior na faixa etária de $9 \mid-12$ meses, e 2,5\% do total de lactentes não consumiram nenhuma fonte láctea. Em relação aos não amamentados, $4,7 \%$ não consumiram outros tipos de leite. O percentual de lactentes que usavam mamadeira ou chuquinha foi superior naqueles com $9 \mid-12$ meses, e a proporção dos que usavam chupeta foi maior naqueles com $6 \mid-8,9$ meses. Bicos artificiais foram mais utilizados por lactentes não amamentados. Observou-se maiores preva- 
Tabela 1. Distribuição e proporção de lactentes paulistas de seis a 11,9 meses de idade, segundo suas características e características das mães. Estado de São Paulo, 2008.

\begin{tabular}{|c|c|c|}
\hline Variável & $\mathbf{N}$ & $\%$ \\
\hline \multicolumn{3}{|l|}{ Faixa etária do lactente } \\
\hline $6 \mid-7$ meses & 2649 & 18,5 \\
\hline $7 \mid-8$ meses & 2371 & 16,5 \\
\hline $8 \mid-9$ meses & 2288 & 16,0 \\
\hline $9 \mid-10$ meses & 2228 & 15,5 \\
\hline $10 \mid-11$ meses & 2244 & 15,7 \\
\hline $11 \mid-12$ meses & 2547 & 17,8 \\
\hline \multicolumn{3}{|l|}{ Sexo } \\
\hline Masculino & 7200 & 50,3 \\
\hline Feminino & 7127 & 49,7 \\
\hline \multicolumn{3}{|l|}{ Peso ao nascer } \\
\hline$<2500 \mathrm{~g}$ & 1233 & 9,2 \\
\hline $2500 \mathrm{~g}$ e mais & 12189 & 90,8 \\
\hline \multicolumn{3}{|l|}{ Tipo de parto } \\
\hline Cesárea & 8345 & 59,0 \\
\hline Vaginal & 5800 & 41,0 \\
\hline \multicolumn{3}{|l|}{$\begin{array}{l}\text { Local de acompanhamento } \\
\text { ambulatorial }\end{array}$} \\
\hline Particular & 5310 & 38,6 \\
\hline $\begin{array}{l}\text { Unidade Básica de Saúde } \\
\text { tradicional }\end{array}$ & 5061 & 36,8 \\
\hline PACS/ PSF* & 3017 & 22,0 \\
\hline Outros locais públicos & 354 & 2,6 \\
\hline \multicolumn{3}{|l|}{ Faixa etária materna } \\
\hline$<20$ anos & 1508 & 12,9 \\
\hline $20 \mid-35$ anos & 8754 & 74,8 \\
\hline 35 anos e mais & 1438 & 12,3 \\
\hline \multicolumn{3}{|l|}{ Paridade } \\
\hline Primípara & 5924 & 51,0 \\
\hline Multípara & 5690 & 49,0 \\
\hline \multicolumn{3}{|l|}{ Situação de trabalho } \\
\hline Trabalha fora & 3722 & 32,7 \\
\hline Não trabalha fora & 7663 & 67,3 \\
\hline \multicolumn{3}{|l|}{ Escolaridade materna } \\
\hline$\leq 8$ anos de estudo & 3767 & 32,6 \\
\hline $9 \mid-12$ anos de estudo & 6057 & 52,4 \\
\hline 12 anos de estudo e mais & 1733 & 15,0 \\
\hline
\end{tabular}

lências de consumo de mingau, água, fruta, papa salgada, carne e alimentos não saudáveis na faixa etária superior e nos lactentes não amamentados, e quase $80 \%$ dos lactentes paulistas consumiram algum tipo de alimento não saudável.

Os indicadores de consumo são apresentados na Tabela 4. A maioria dos lactentes apresentou classificação negativa para diversidade mínima
$(68,2 \%)$, adequação da dieta $(72,7 \%)$ ou dieta minimamente aceitável $(71,1 \%)$.

\section{Discussão}

O consumo de mingau, água, fruta, papa salgada, carne e de alimentos não saudáveis foi mais prevalente nos lactentes mais velhos e naqueles não amamentados. Em relação aos indicadores de consumo, a menor parte dos lactentes apresentou classificação positiva em relação à diversidade mínima, adequação da dieta e dieta minimamente aceitável.

No presente estudo, 91\% dos lactentes nasceram com peso $\geq 2500$ g, e quase $13 \%$ das mães tinham menos de 20 anos; achados semelhantes foram encontrados para todo o estado de São Paulo, em 2008: 91,0\% das crianças nasceram com peso $\geq 2500 \mathrm{~g}$, e $16,7 \%$ das mulheres tinham menos de $20 \operatorname{anos}^{20}$. Em relação às características municipais, metade dos municípios paulistas que participaram da PPAM apresentava grau de urbanização superior a 90\%, e a maioria tinha cobertura inferior a 5\% do SISVAN. Esses achados também vão ao encontro do observado para o estado, que em 2008 apresentava grau de urbanização de $95,2 \%{ }^{14}$ e cobertura do SISVAN de $1,9 \%{ }^{10}$. Tais dados indicam que, embora a PPAM não tenha sido desenhada para representar os municípios paulistas, é possível generalizar os achados do presente estudo para o estado como um todo.

Enes et al. ${ }^{16}$, ao avaliarem a cobertura do SISVAN em 65 municípios de São Paulo, encontraram resultado melhor que o do presente estudo: mais da metade deles apresentou cobertura entre $5 \%$ e $10 \%$ em 2010. Pode-se afirmar que, apesar dos esforços adotados pelo governo, a vigilância alimentar e nutricional no estado de São Paulo ainda deixa a desejar, comprometendo o aproveitamento do Sistema na (re)formulação de políticas de alimentação e nutrição ${ }^{16}$.

Parte considerável dos municípios avaliados apresentou cobertura da ESF inferior a 30\%, valor considerado incipiente: estudo realizado em municípios brasileiros verificou associação negativa entre cobertura do Programa Saúde da Família - atualmente denominado ESF - e mortalidade infantil ${ }^{21}$. Porém, mais de $1 / 3$ dos municípios apresentaram cobertura superior a $70 \%$ do PACS. Os agentes comunitários de saúde são fundamentais para o sucesso de atividades de incentivo à alimentação infantil, por estarem mais envolvidos com a comunidade em suas funções habituais ${ }^{22}$. 
Tabela 2. Características dos 76 municípios estudados. Estado de São Paulo, 2008.

\begin{tabular}{|c|c|c|}
\hline Variável & $\mathbf{N}$ & $\%$ \\
\hline \multicolumn{3}{|l|}{ Grau de urbanização } \\
\hline$<25 \%$ & 0 & 0,0 \\
\hline $25 \mid-50 \%$ & 2 & 2,6 \\
\hline $50 \mid-75 \%$ & 9 & 11,8 \\
\hline $75 \mid-90 \%$ & 27 & 35,5 \\
\hline $90 \%$ e mais & 38 & 50,0 \\
\hline \multicolumn{3}{|c|}{$\begin{array}{l}\text { Porte populacional (número de } \\
\text { habitantes) }\end{array}$} \\
\hline$<15$ mil & 29 & 38,2 \\
\hline 15|-100 mil & 31 & 40,8 \\
\hline 100 mil|-1 milhão & 13 & 17,1 \\
\hline$\geq 1$ milhão & 3 & 3,9 \\
\hline \multicolumn{3}{|c|}{$\begin{array}{l}\text { Cobertura da Estratégia Saúde da } \\
\text { Família (ESF) }\end{array}$} \\
\hline$<15 \%$ & 25 & 32,9 \\
\hline $15 \mid-30 \%$ & 12 & 15,8 \\
\hline $30 \mid-50 \%$ & 12 & 15,8 \\
\hline $50 \mid-70 \%$ & 6 & 7,9 \\
\hline $70 \%$ e mais & 21 & 27,6 \\
\hline \multirow{2}{*}{\multicolumn{3}{|c|}{$\begin{array}{l}\text { Cobertura do Programa Agentes } \\
\text { Comunitários de Saúde (PACS) }\end{array}$}} \\
\hline & & \\
\hline$<15 \%$ & 19 & 25,0 \\
\hline $15 \mid-30 \%$ & 14 & 18,4 \\
\hline $30 \mid-50 \%$ & 11 & 14,5 \\
\hline $50 \mid-70 \%$ & 7 & 9,2 \\
\hline $70 \%$ e mais & 25 & 32,9 \\
\hline \multicolumn{3}{|c|}{$\begin{array}{l}\text { Cobertura do Sistema de Vigilância } \\
\text { Alimentar e Nutricional (SISVAN) }\end{array}$} \\
\hline$<5 \%$ & 66 & 86,8 \\
\hline $5 \mid-10 \%$ & 7 & 9,2 \\
\hline $10 \mid-15 \%$ & 2 & 2,6 \\
\hline $15 \% \mid-20 \%$ & 1 & 1,3 \\
\hline \multicolumn{3}{|c|}{$\begin{array}{l}\text { Índice Paulista de } \\
\text { Responsabilidade Social (IPRS) }\end{array}$} \\
\hline 1 e 2 (alta riqueza) & 25 & 32,9 \\
\hline 3,4 e 5 (baixa riqueza) & 51 & 67,1 \\
\hline
\end{tabular}

A prevalência de amamentação entre lactentes paulistas foi de 56,1\%. O estudo de Saldiva et al..$^{23}$, que avaliou lactentes da mesma faixa etária de 136 municípios do estado de São Paulo em 2004, encontrou prevalência de aleitamento materno de 50\%, mostrando que houve melhora neste indicador. Comparando as duas faixas etárias estudadas, verificou-se que lactentes mais velhos apresentaram prevalência inferior de aleitamento materno: 50,6\%. A mesma preva- lência foi encontrada pela Pesquisa Nacional de Saúde (PNS) de 2013 para esta faixa etária ${ }^{24}$; já a II PPAM constatou que $58,7 \%$ de nove a 11,9 meses estavam em aleitamento materno ${ }^{13}$. A menor prevalência de aleitamento materno em lactentes mais velhos pode ser atribuída ao fato de receberem outros tipos de alimentos com maior frequência - os quais, muitas vezes, acabam por substituir o leite materno. Ressalta-se que a recomendação é de que as crianças devem ser amamentadas até os dois anos de idade ou mais, pois mesmo com a introdução de outros alimentos, o leite materno continua nutrindo a criança e protegendo-a contra doenças ${ }^{1,2}$.

Em relação ao consumo de lácteos, observouse que $70,2 \%$ dos lactentes paulistas consumiram outros tipos de leite, e que este consumo foi maior entre lactentes mais velhos; verificou-se, ainda, que 2,5\% dos lactentes não consumiram leite materno nem outros tipos de leite no dia anterior. Saldiva et al. ${ }^{23}$ verificaram prevalência superior de consumo de outros tipos de leite (77\%), e que o consumo dos mesmos foi maior com o aumento da idade. Estudo conduzido no município de São Paulo com lactentes menores de um ano verificou que $74,8 \%$ recebiam outros tipos de leite ${ }^{25}$. No estado da Paraíba, 85,9\% dos lactentes entre seis e 11,9 meses consumiram refeições lácteas ${ }^{26}$. Destaca-se que a falta de lácteos na dieta das crianças é preocupante, pois esses alimentos fornecem proteínas e cálcio, fundamental para o desenvolvimento ósseo ${ }^{1}$.

Aproximadamente 10\% dos lactentes estudados não consumiram água, e cerca de 1/5 consumiram chá. $\mathrm{O}$ consumo de ambos foi mais frequente em lactentes não amamentados, achado semelhante ao de Vieira et al. ${ }^{27}$. em Feira de Santana (Bahia). Estudo conduzido em Botucatu (São Paulo) verificou, entre lactentes de seis a 9,9 meses amamentados, que $94,8 \%$ consumiram água e $22,6 \%$ consumiram chá ${ }^{28}$. O baixo consumo de água merece atenção, pois o Ministério da Saúde recomenda que a criança receba água nos intervalos das refeições após início da alimentação complementar; ainda, o consumo de chás não é indicado pelo baixo valor nutricional e porque algumas de suas substâncias podem irritar a mucosa gástrica dos lactentes ${ }^{1}$.

No presente estudo, 3/4 dos lactentes usaram mamadeira ou chuquinha e aproximadamente metade utilizou chupeta. Ainda, quase todos os lactentes não amamentados usavam algum bico artificial. Observa-se pior quadro no uso de bicos artificiais no estado de São Paulo quando comparado ao Sudeste e ao país: de acordo com a II 
Tabela 3. Distribuição e proporção de características de consumo alimentar e do uso de bicos artificiais entre lactentes de seis a 11,9 meses de idade, segundo faixa etária do lactente e situação de aleitamento materno. Estado de São Paulo, 2008.

\begin{tabular}{|c|c|c|c|c|c|c|c|c|c|c|c|c|}
\hline \multirow[t]{2}{*}{ Variável } & \multicolumn{2}{|c|}{$6 \mid-9$ meses } & \multicolumn{2}{|c|}{$9 \mid-12$ meses } & \multirow[t]{2}{*}{$\mathbf{p}$} & \multicolumn{2}{|c|}{$\begin{array}{c}\text { Lactentes } \\
\text { amamentados }\end{array}$} & \multicolumn{2}{|c|}{$\begin{array}{l}\text { Lactentes não } \\
\text { amamentados }\end{array}$} & \multirow[t]{2}{*}{$\mathbf{p}$} & \multicolumn{2}{|c|}{ Total } \\
\hline & $\mathbf{N}$ & $\%$ & $\mathbf{N}$ & $\%$ & & $\mathbf{N}$ & $\%$ & $\mathbf{N}$ & $\%$ & & $\mathbf{N}$ & $\%$ \\
\hline Aleitamento materno & & & & & 0,000 & & & & & NA & & \\
\hline Sim & 4443 & 61,4 & 3512 & 50,6 & & NA & NA & NA & NA & & 7955 & 56,1 \\
\hline Não & 2794 & 38,6 & 3433 & 49,4 & & $\mathrm{NA}$ & NA & NA & NA & & 6227 & 43,9 \\
\hline Consumo de leite• & & & & & 0,000 & & & & & 0,000 & & \\
\hline Sim & 4792 & 66,2 & 5170 & 74,4 & & 3970 & 50,3 & 5889 & 95,3 & & 9962 & 70,2 \\
\hline Não & 2443 & 33,8 & 1782 & 25,6 & & 3920 & 49,7 & 291 & 4,7 & & 4225 & 29,8 \\
\hline Consumo de lácteos $^{\Delta}$ & & & & & 0,015 & & & & & & & \\
\hline Sim & 7138 & 97,8 & 6809 & 97,2 & & 7955 & 100,0 & 5889 & 94,6 & & 13947 & 97,5 \\
\hline Não & 157 & 2,2 & 195 & 2,8 & & 0 & 0,0 & 338 & 5,4 & & 352 & 2,5 \\
\hline $\begin{array}{l}\text { Uso de mamadeira ou } \\
\text { chuquinha }\end{array}$ & & & & & 0,000 & & & & & 0,000 & & \\
\hline Sim & 5365 & 73,7 & 5329 & 76,2 & & 4588 & 57,9 & 6004 & 96,6 & & 10694 & 74,9 \\
\hline Não & 1919 & 26,3 & 1664 & 23,8 & & 3335 & 42,1 & 209 & 3,4 & & 3583 & 25,1 \\
\hline Uso de chupeta & & & & & 0,000 & & & & & 0,000 & & \\
\hline Sim & 3672 & 50,5 & 3253 & 46,5 & & 2360 & 29,8 & 4495 & 72,4 & & 6925 & 48,5 \\
\hline Não & 3604 & 49,5 & 3739 & 53,5 & & 5557 & 70,2 & 1714 & 27,6 & & 7343 & 51,5 \\
\hline Uso de bicos artificiais" & & & & & 0,303 & & & & & 0,000 & & \\
\hline Sim & 5777 & 79,2 & 5597 & 79,8 & & 5168 & 65,1 & 6098 & 98,0 & & 11374 & 79,5 \\
\hline Não & 1522 & 20,8 & 1413 & 20,2 & & 2774 & 34,9 & 126 & 2,0 & & 2935 & 20,5 \\
\hline $\begin{array}{l}\text { Consumo de mingau } \\
\text { (doce ou salgado) }\end{array}$ & & & & & 0,029 & & & & & 0,000 & & \\
\hline Sim & 2546 & 35,5 & 2569 & 37,3 & & 2516 & 32,1 & 2543 & 41,6 & & 5115 & 36,4 \\
\hline Não & 4628 & 64,5 & 4325 & 62,7 & & 5320 & 67,9 & 3573 & 58,4 & & 8953 & 63,6 \\
\hline Consumo de água & & & & & 0,000 & & & & & 0,000 & & \\
\hline Sim & 6425 & 89,0 & 6488 & 93,4 & & 7014 & 88,7 & 5809 & 94,4 & & 12913 & 91,1 \\
\hline Não & 798 & 11,0 & 457 & 6,6 & & 892 & 11,3 & 345 & 5,6 & & 1255 & 8,9 \\
\hline Consumo de chá & & & & & 0,667 & & & & & 0,000 & & \\
\hline Sim & 1484 & 20,6 & 1408 & 20,4 & & 1460 & 18,6 & 1399 & 22,8 & & 2892 & 20,5 \\
\hline Não & 5705 & 79,4 & 5511 & 79,6 & & 6401 & 81,4 & 4748 & 77,2 & & 11216 & 79,5 \\
\hline $\begin{array}{l}\text { Consumo de fruta (em } \\
\text { pedaço ou amassada) }\end{array}$ & & & & & 0,000 & & & & & 0,000 & & \\
\hline Sim & 5282 & 73,2 & 5404 & 78,2 & & 5767 & 73,3 & 4820 & 78,6 & & 10686 & 75,7 \\
\hline Não & 1931 & 26,8 & 1507 & 21,8 & & 2106 & 26,7 & 1309 & 21,4 & & 3438 & 24,3 \\
\hline $\begin{array}{l}\text { Consumo de suco/ } \\
\text { água de coco natural }\end{array}$ & & & & & 0,661 & & & & & 0,000 & & \\
\hline Sim & 4759 & 66,3 & 4616 & 66,7 & & 5008 & 63,9 & 4285 & 69,8 & & 9375 & 66,5 \\
\hline Não & 2415 & 33,7 & 2306 & 33,3 & & 2827 & 36,1 & 1854 & 30,2 & & 4721 & 33,5 \\
\hline $\begin{array}{l}\text { Consumo de papa } \\
\text { salgada }\end{array}$ & & & & & 0,000 & & & & & 0,000 & & \\
\hline Sim & 6402 & 88,6 & 6594 & 95,1 & & 7055 & 89,6 & 5823 & 94,5 & & 12996 & 91,8 \\
\hline Não & 827 & 11,4 & 340 & 4,9 & & 822 & 10,4 & 339 & 5,5 & & 1167 & 8,2 \\
\hline Consumo de carne & & & & & 0,000 & & & & & 0,000 & & \\
\hline Sim & 4430 & 69,4 & 5360 & 81,4 & & 5198 & 73,8 & 4504 & 77,6 & & 9790 & 75,5 \\
\hline Não & 1950 & 30,6 & 1226 & 18,6 & & 1845 & 26,2 & 1302 & 22,4 & & 3176 & 24,5 \\
\hline Consumo de feijão & & & & & 0,000 & & & & & 0,327 & & \\
\hline Sim & 4640 & 72,6 & 5587 & 84,9 & & 5533 & 78,5 & 4602 & 79,2 & & 10227 & 78,9 \\
\hline Não & 1752 & 27,4 & 991 & 15,1 & & 1513 & 21,5 & 1206 & 20,8 & & 2743 & 21,1 \\
\hline
\end{tabular}


Tabela 3. Distribuição e proporção de características de consumo alimentar e do uso de bicos artificiais entre lactentes de seis a 11,9 meses de idade, segundo faixa etária do lactente e situação de aleitamento materno. Estado de São Paulo, 2008.

\begin{tabular}{|c|c|c|c|c|c|c|c|c|c|c|c|c|}
\hline \multirow[t]{2}{*}{ Variável } & \multicolumn{2}{|c|}{$6 \mid-9$ meses } & \multicolumn{2}{|c|}{$9 \mid-12$ meses } & \multirow[t]{2}{*}{ p } & \multicolumn{2}{|c|}{$\begin{array}{c}\text { Lactentes } \\
\text { amamentados }\end{array}$} & \multicolumn{2}{|c|}{$\begin{array}{l}\text { Lactentes não } \\
\text { amamentados }\end{array}$} & \multirow[t]{2}{*}{$\mathbf{p}$} & \multicolumn{2}{|c|}{ Total } \\
\hline & $\mathbf{N}$ & $\%$ & $\mathbf{N}$ & $\%$ & & $\mathbf{N}$ & $\%$ & $\mathbf{N}$ & $\%$ & & $\mathbf{N}$ & $\%$ \\
\hline $\begin{array}{l}\text { Consumo de verduras } \\
\text { e/ou legumes }\end{array}$ & & & & & 0,013 & & & & & 0,759 & & \\
\hline Sim & 5873 & 91,8 & 5978 & 90,6 & & 6436 & 91,1 & 5309 & 91,3 & & 11851 & 91,2 \\
\hline Não & 525 & 8,2 & 623 & 9,4 & & 629 & 8,9 & 509 & 8,7 & & 1148 & 8,8 \\
\hline $\begin{array}{l}\text { Consumo de suco } \\
\text { industrializado ou água } \\
\text { de coco em caixinha }\end{array}$ & & & & & 0,000 & & & & & 0,009 & & \\
\hline Sim & 839 & 11,6 & 1402 & 20,2 & & 1183 & 15,0 & 1026 & 16,6 & & 2241 & 15,8 \\
\hline Não & 6406 & 88,4 & 5551 & 79,8 & & 6713 & 85,0 & 5154 & 83,4 & & 11957 & 84,2 \\
\hline $\begin{array}{l}\text { Consumo de } \\
\text { refrigerante }\end{array}$ & & & & & 0,000 & & & & & 0,433 & & \\
\hline Sim & 469 & 6,5 & 1078 & 15,5 & & 844 & 10,7 & 688 & 11,1 & & 1547 & 10,9 \\
\hline Não & 6790 & 93,5 & 5877 & 84,5 & & 7054 & 89,3 & 5510 & 88,9 & & 12667 & 89,1 \\
\hline Consumo de café & & & & & 0,000 & & & & & 0,276 & & \\
\hline $\operatorname{Sim}$ & 440 & 6,1 & 685 & 9,7 & & 606 & 7,7 & 506 & 8,2 & & 1125 & 7,9 \\
\hline Não & 6808 & 93,9 & 6263 & 90,1 & & 7284 & 92,3 & 5680 & 91,8 & & 13071 & 92,1 \\
\hline $\begin{array}{l}\text { Consumo de alimentos } \\
\text { adoçados }^{¥}\end{array}$ & & & & & 0,000 & & & & & 0,000 & & \\
\hline Sim & 2826 & 39,1 & 3292 & 47,4 & & 3104 & 39,3 & 2952 & 47,8 & & 6118 & 43,1 \\
\hline Não & 4410 & 60,9 & 3656 & 52,6 & & 4787 & 60,7 & 3221 & 52,2 & & 8066 & 56,9 \\
\hline $\begin{array}{l}\text { Consumo de bolacha, } \\
\text { biscoito ou salgadinho }\end{array}$ & & & & & 0,000 & & & & & 0,000 & & \\
\hline Sim & 4139 & 57,3 & 5405 & 78,0 & & 5168 & 65,6 & 4297 & 69,8 & & 9544 & 67,4 \\
\hline Não & 3085 & 42,7 & 1526 & 22,0 & & 2709 & 34,4 & 1860 & 30,2 & & 4611 & 32,6 \\
\hline $\begin{array}{l}\text { Consumo de alimentos } \\
\text { não saudáveis }{ }^{\epsilon}\end{array}$ & & & & & 0,000 & & & & & 0,000 & & \\
\hline Sim & 5264 & 72,3 & 6117 & 87,5 & & 6119 & 77,2 & 5165 & 83,1 & & 11381 & 79,8 \\
\hline Não & 2018 & 27,7 & 872 & 12,5 & & 1812 & 22,8 & 1051 & 16,9 & & 2890 & 20,2 \\
\hline
\end{tabular}

PPAM, 63,8\% dos menores de um ano usaram mamadeira no Sudeste e $58,4 \%$ no país; o uso de chupeta nesta região foi de $50,3 \%$ - semelhante ao presente estudo - e no Brasil foi de $42,6 \%{ }^{13}$. Mamadeiras fazem com que a criança degluta ar e tenha desconfortos abdominais; ademais, bicos artificiais aumentam o risco de problemas ortodônticos e fonoaudiológicos ${ }^{1}$. Oliveira et $\mathrm{al}^{19}$. consideraram a utilização de bicos artificiais como atributo de ausência de inocuidade microbiológica pela dificuldade de se manterem limpos, podendo ser veículos de contaminação.

Em comparação aos achados do estudo realizado no estado de São Paulo em 2004, verificou-se que o consumo de frutas diminuiu, mas o de feijão e carne aumentou: para aquele ano, $87 \%$ dos lactentes haviam consumido frutas, 58\% feijão e $36 \%$ carne $^{23}$. Estudo de Bortolini et al. ${ }^{6}$ sobre consumo alimentar de 4322 crianças, com idade entre seis a 59 meses, investigadas na Pesquisa Nacional de Demografia e Saúde 2006-2007, encontrou que a região Sudeste foi uma das que apresentou maiores percentuais do consumo destes alimentos. $\mathrm{O}$ consumo de frutas, carnes e feijão foi maior entre lactentes paulistas mais velhos; porém, semelhante a outro estudo ${ }^{25}$, verificou-se o contrário para verduras e/ ou legumes. É provável que lactentes mais velhos consumam menos verduras e legumes 
Tabela 4. Distribuição e proporção de indicadores de consumo alimentar entre lactentes de seis a 11,9 meses de idade. Estado de São Paulo, 2008.

\begin{tabular}{|c|c|c|}
\hline Variável & $\mathbf{N}$ & $\%$ \\
\hline \multicolumn{3}{|c|}{$\begin{array}{l}\text { Consumo de alimentos ricos em } \\
\text { ferro }^{\star}\end{array}$} \\
\hline Sim & 12282 & 94,2 \\
\hline Não & 760 & 5,8 \\
\hline \multicolumn{3}{|l|}{ Alimentação oportuna $^{\dagger}$} \\
\hline Sim & 4973 & 69,2 \\
\hline Não & 2215 & 30,8 \\
\hline \multicolumn{3}{|c|}{$\begin{array}{l}\text { Consistência adequada da papa } \\
\text { salgada }^{\ddagger}\end{array}$} \\
\hline Sim & 10681 & 83,2 \\
\hline Não & 2155 & 16,8 \\
\hline \multicolumn{3}{|l|}{ Densidade energética ${ }^{\S}$} \\
\hline $\operatorname{Sim}$ & 7811 & 63,1 \\
\hline Não & 4574 & 36,9 \\
\hline \multicolumn{3}{|c|}{ Diversidade mínima da dieta" } \\
\hline Sim & 4546 & 31,8 \\
\hline Não & 9756 & 68,2 \\
\hline \multicolumn{3}{|l|}{ Adequação da dieta" } \\
\hline Sim & 3909 & 27,3 \\
\hline Não & 10393 & 72,7 \\
\hline \multicolumn{3}{|c|}{ Dieta minimamente aceitável ${ }^{£}$} \\
\hline Sim & 4132 & 28,9 \\
\hline Não & 10178 & 71,1 \\
\hline \multicolumn{3}{|c|}{ 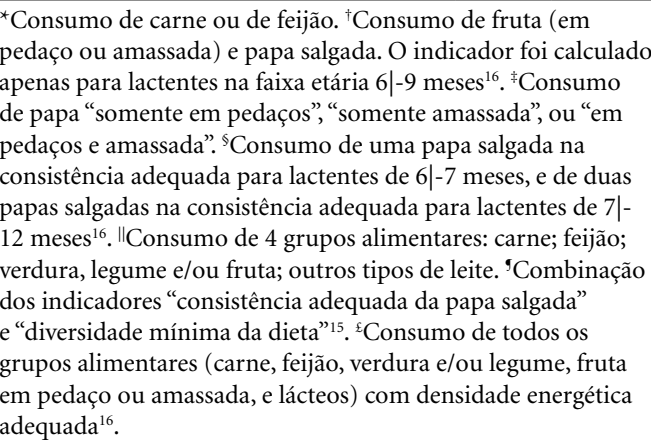 } \\
\hline
\end{tabular}

porque já começaram a receber alimentação da família ao invés da refeição preparada somente para eles, e o consumo destes alimentos pelos brasileiros, geralmente, é insatisfatório.

O consumo de verduras e/ ou legumes não diferiu entre lactentes amamentados e não amamentados. Já o consumo de frutas, suco/ água de coco natural, papa salgada e carne foi maior entre não amamentados. O estudo de Botucatu observou que, entre lactentes amamentados de seis a 9,9 meses, $76,6 \%$ haviam consumido frutas, $41,6 \%$ carnes e $43,5 \%$ feijão ${ }^{28}$. É preocupante o fato de aproximadamente $10 \%$ dos lactentes paulistas não terem recebido papa salgada nem ver- duras e/ ou legumes, e de mais de $20 \%$ não terem consumido frutas, carne ou feijão no dia anterior. O passo 3 do Guia alimentar para crianças menores de dois anos preconiza o consumo de cereais, tubérculos, carnes, leguminosas, frutas e legumes na alimentação complementar, informando que o consumo de tais alimentos promove maior ingestão de vitaminas, fibras e ferro ${ }^{1}$.

Dados da II PPAM mostram situação melhor do Brasil comparada ao estado de São Paulo em relação ao consumo de alimentos não saudáveis: entre lactentes de 9-11,9 meses, 8,7\% haviam consumido café, $11,6 \%$ refrigerante e $71,7 \%$ bolacha, biscoito ou salgadinho ${ }^{13}$. Bortolini et al. ${ }^{6}$ encontraram consumo diário de bolachas e biscoitos em $46,3 \%$, de salgadinhos em $8,5 \%$ e de refrigerante em $22,1 \%$ das crianças, e observaram que o consumo de doces e refrigerantes foi mais frequente no Sudeste em comparação às demais regiões. A PNS verificou que, entre as crianças menores de dois anos, 60,8\% consumiram bolacha, biscoito ou bolo, e que 32,3\% ingeriram refrigerante ou suco artificial ${ }^{24}$. O passo 8 do Guia alimentar destaca que o consumo destes alimentos é prejudicial à saúde da criança por associarem-se à anemia, ao excesso de peso e às alergias alimentares, além de conterem sódio, açúcar, gorduras e aditivos artificiais em excesso, recomendando evitá-los no primeiro ano de vida ${ }^{1}$.

A maioria dos lactentes paulistas consumiu algum alimento rico em ferro; entretanto, 5,8\% da população estudada não consumiram carne nem feijão no dia anterior à entrevista. Estudo conduzido em Belo Horizonte (Minas Gerais) verificou que a ingestão de diversos nutrientes avaliados em lactentes estava acima de $100 \%$ da Ingestão Diária de Referência, com exceção do ferro $^{29}$. A deficiência deste mineral é considerada alarmante, pois pode levar à anemia ${ }^{1}$.

A prevalência de alimentação complementar oportuna entre lactentes paulistas de seis a 8,9 meses foi de $69,2 \%$. Apesar deste valor ser superior ao encontrado por outros estudos - para a mesma faixa etária, Saldiva et al. ${ }^{23}$ observaram prevalência de $48 \%$, Palmeira et al. ${ }^{26}$. verificaram frequência de 27,1\% e estudo realizado em Volta Redonda (Rio de Janeiro) constatou percentual de $50,0 \%{ }^{30}$, este achado sugere que quase $1 / 3$ dos lactentes paulistas apresentam consumo alimentar insatisfatório logo no início da alimentação complementar.

No presente estudo, $83,2 \%$ dos lactentes consumiram papa salgada em consistência adequada, e $63,1 \%$ foram classificados de forma satisfatória para o indicador "densidade energética". De forma 
semelhante, o estudo de Volta Redonda observou que $83,7 \%$ dos lactentes receberam preparações em pedaços ou amassadas ${ }^{30}$. Preparações liquidificadas ou peneiradas não estimulam a mastigação e costumam apresentar menor concentração de energia e nutrientes, o que se agrava quando ofertadas em frequência insuficiente ${ }^{1}$.

Apenas 31,8\% dos lactentes estudados apresentaram diversidade mínima da dieta, e as prevalências de adequação da dieta e de dieta minimamente aceitável foram inferiores a $30 \%$. Estudo de base populacional realizado no Nepal detectou que lactentes de seis a onze meses apresentaram os piores indicadores de diversidade da dieta, frequência mínima da dieta (o qual corresponde ao indicador "densidade energética" do presente estudo) e dieta minimamente aceitável ${ }^{31}$. Estes indicadores refletem variedade, consistência e frequência da dieta, aspectos fundamentais para adequada oferta energética e nutricional ${ }^{1,17}$.

Dentre as limitações do estudo, destaca-se o delineamento transversal, que não permite estabelecer a procedência temporal necessária para identificação de relações causais, e o fato das informações referentes à alimentação dos lactentes referirem-se apenas ao dia anterior ao inquérito, o que impossibilita analisar com que frequência os alimentos avaliados foram consumidos. Por outro lado, os aspectos inovadores do presente estudo foram a caracterização da população de lactentes, de suas mães e dos municípios paulistas incluídos na PPAM, e a exploração de uma série de dados referentes à alimentação da população estudada e a obtenção de importantes indicadores de consumo alimentar. Destaca-se também que este é o estudo epidemiológico mais abrangente e recente realizado no estado de São Paulo que tem como foco a alimentação de lactentes, sendo a base de dados mais atual para avaliar o consumo alimentar desta população.

No presente estudo foram encontradas maiores prevalências de consumo de mingau, água, fruta, papa salgada, carne e de alimentos não saudáveis entre lactentes paulistas mais velhos e não amamentados. Observou-se ainda prevalências insatisfatórias para diversos indicadores calculados, sugerindo que a minoria dos lactentes está recebendo alimentos complementares necessários em quantidade, frequência e consistência adequadas para seu pleno desenvolvimento. Estas informações podem servir como subsídios para direcionar ações sobre alimentação infantil.

\section{Colaboradoras}

A Passanha, MHDA Benício e SI Venancio participaram substancialmente da elaboração deste manuscrito e todas revisaram a versão final do documento, aprovando-a para submissão. 


\section{Referências}

1. Brasil. Ministério da Saúde (MS). Secretaria de Atenção à Saúde, Departamento de Atenção Básica. Dez passos para uma alimentação saudável: guia alimentar para menores de dois anos - um guia para o profissional da saúde na atenção básica. 2a ed. Brasília: MS; 2010.

2. World Health Organization (WHO). The optimal duration of exclusive breastfeeding: a systematic review. Geneva: WHO; 2002.

3. Pan American Health Organization (PAHO), World Health Organization (WHO). Guiding principles for complementary feeding of the breastfed child. Washington: PAHO; 2003.

4. World Health Organization (WHO). Global strategy for infant and young child feeding. Geneva: WHO; 2003.

5. Brasil. Ministério da Saúde (MS). Secretaria de Atenção à Saúde, Departamento de Atenção Básica. Saúde da criança: aleitamento materno e alimentação complementar. $2^{\mathrm{a}}$ ed. Brasília: MS; 2015.

6. Bortolini GA, Gubert MB, Santos LMP. Consumo alimentar entre crianças brasileiras com idade de 6 a 59 meses. Cad Saude Publica 2012; 28(9):1759-1771.

7. Jordão RE, Bernardi JLD, Filho AAB. Introdução alimentar e anemia em lactentes do município de Campinas (SP). Rev Paul Pediatr 2009; 27(4):381-388.

8. Partearroyo T, Campayo ES, Moreiras GV. Sugar at different stages in life; from childhood to old age. Nutr Hosp 2013; 28(Supl. 4):40-47.

9. Rose CM, Birch LL, Savage JS. Dietary patterns in infancy are associated with child diet and weight outcomes at 6 years. Int J Obes 2017; 41(5):783-788.

10. Sistema de Vigilância Alimentar e Nutricional (SISVAN). Módulo gerador de relatórios [Internet]. 2018 [citado 2018 Jan 28]. Disponível em: http://dabsistemas.saude.gov.br/sistemas/sisvan/relatorios_publicos/relatorios.php

11. Brasil. Ministério da Saúde (MS). Secretaria de Atenção à Saúde, Departamento de Ações Programáticas e Estratégicas. Pesquisa de Prevalência de Aleitamento Materno em Municípios Brasileiros. Brasília: MS; 2010

12. Venâncio SI, Saldiva SRDM, Castro ALS, Gouveia AGC, Santana AC, Pinto JCC, Escuder MML. Projeto Amamentação e Municípios: a trajetória de implantação de uma estratégia para a avaliação e monitoramento das práticas de alimentação infantil no Estado de São Paulo, no período de 1998-2008. BEPA - Boletim Epidemiológico Paulista 2010; 7(83):4-15.

13. Brasil. Ministério da Saúde (MS). Secretaria de Atenção à Saúde, Departamento de Ações Programáticas e Estratégicas. II Pesquisa de Prevalência de Aleitamento Materno nas Capitais Brasileiras e Distrito Federal. Brasília: MS; 2009.

14. Fundação Sistema Estadual de Análise de Dados (SEADE). Portal de Estatísticas do Estado de São Paulo [Internet]. 2018 [citado 2018 Jan 28]. Disponível em: http://www.seade.gov.br

15. Governo do Estado de São Paulo. Portal do Governo [Internet]. 2018 [citado 2018 Jan 28]. Disponível em: http://www.saopaulo.sp.gov.br/conhecasp/

16. Enes CC, Loiola H, Oliveira MRM. Cobertura populacional do Sistema de Vigilância Alimentar e Nutricional no Estado de São Paulo, Brasil. Cien Saude Colet 2014; 19(5):1543-1551.
17. World Health Organization (WHO). Indicators for assessing infant and young child feeding practices - part 1: definitions. Geneva: WHO; 2008.

18. Oliveira DA, Castro IRR, Jaime PC. Complementary feeding patterns in the first year of life in the city of Rio de Janeiro, Brazil: time trends from 1998 to 2008. Cad Saude Publica 2014; 30(8):1755-1764.

19. Oliveira JM, Castro IRR, Silva GB, Venâncio SI, Saldiva SRDM. Avaliação da alimentação complementar nos dois primeiros anos de vida: proposta de indicadores e de instrumento. Cad Saude Publica 2015; 31(2):377-394.

20. Banco de Dados do Sistema Único de Saúde (DATASUS). Informações de Saúde (TABNET) [Internet]. 2018 [citado 2018 Jan 28]. Disponível em: http:// www2.datasus.gov.br/DATASUS/index.php?area $=02$

21. Aquino R, Oliveira NF, Barreto ML. Impact of the Family Health Program on Infant Mortality in Brazilian Municipalities. Am J Public Health 2009; 99(1):8793.

22. Caldeira AP, Fagundes GC, Aguiar GN. Intervenção educacional em equipes do Programa de Saúde da Família para promoção da amamentação. Rev Saude Publica 2008; 42(6):1027-1033.

23. Saldiva SRDM, Escuder MM, Mondini L, Levy RB, Venâncio SI. Práticas alimentares de crianças de 6 a 12 meses e fatores maternos associados. J Pediatr (Rio J) 2007; 83(1):53-58.

24. Instituto Brasileiro de Geografia e Estatística (IBGE). Pesquisa Nacional de Saúde 2013: Ciclos de vida - Brasil e grandes regiões. Rio de Janeiro: IBGE; 2015.

25. Souza SB, Szarfarc SC, Souza JMP. Prática alimentar no primeiro ano de vida, em crianças atendidas em centros de saúde escola do município de São Paulo. Rev Nutr 1999; 12(2):167-174.

26. Palmeira PA, Santos SMC, Vianna RPT. Prática alimentar entre crianças menores de dois anos de idade residentes em municípios do semiárido do Estado da Paraíba, Brasil. Rev Nutr 2011; 24(4):553-563.

27. Vieira GO, Silva LR, Vieira TO, Almeida JAG, Cabral VA. Hábitos alimentares de crianças menores de 1 ano amamentadas e não-amamentadas. J Pediatr (Rio J) 2004; 80(5):411-416.

28. Parada CMGL, Carvalhaes MABL, Jamas MT. Complementary feeding practices to children during their first year of life. Rev Latinoam Enferm 2007; 15(2):282-289.

29. Lima DB, Fujimori E, Borges ALV, Silva MMS. Prática alimentar nos dois primeiros anos de vida. Rev Esc Enferm USP 2011; 45(2):1705-1709.

30. Cruz MCC, Almeida JAG, Engstrom EM. Práticas alimentares no primeiro ano de vida de filhos de adolescentes. Rev Nutr 2010; 23(2):201-210.

31. Khanal V, Sauer K, Zhao Y. Determinants of complementary feeding practices among Nepalese children aged 6-23 months: findings from demographic and health survey 2011. BMC Pediatrics 2013; 13:131.

Artigo apresentado em 09/09/2017

Aprovado em 07/06/2018

Versão final apresentada em 09/06/2018 
